\title{
The Hawking evaporation process of rapidly-rotating black holes: an almost continuous cascade of gravitons
}

\author{
Shahar Hod ${ }^{1,2, a}$ \\ ${ }^{1}$ The Ruppin Academic Center, 40250 Emek Hefer, Israel \\ 2 The Hadassah Institute, 91010 Jerusalem, Israel
}

Received: 17 June 2015 / Accepted: 1 July 2015 / Published online: 17 July 2015

(C) The Author(s) 2015. This article is published with open access at Springerlink.com

\begin{abstract}
It is shown that rapidly-rotating Kerr black holes are characterized by the dimensionless ratio $\tau_{\text {gap }} / \tau_{\text {emission }}=$ $O(1)$, where $\tau_{\text {gap }}$ is the average time gap between the emissions of successive Hawking quanta and $\tau_{\text {emission }}$ is the characteristic timescale required for an individual Hawking quantum to be emitted from the black hole. This relation implies that the Hawking cascade from rapidly-rotating black holes has an almost continuous character. Our results correct some inaccurate claims that recently appeared in the literature regarding the nature of the Hawking black-hole evaporation process.
\end{abstract}

\section{Introduction}

Working within the framework of semi-classical general relativity, Hawking [1] has revealed that black holes are actually not completely black. In particular, it was shown [1] that black holes are characterized by thermally distributed emission spectra. ${ }^{1}$ This intriguing finding is certainly one of the most important theoretical predictions of modern physics.

It has recently been pointed out [2] that the Hawking radiation flux out of spherically-symmetric (non-rotating) Schwarzschild black holes is extremely sparse (see [3-5] for earlier discussions of this black-hole property). In particular, denoting by $\tau_{\text {gap }}$ the average time gap between the emissions of successive black-hole quanta, and by $\tau_{\text {emission }}$ the characteristic timescale required for an individual Hawking quantum to be emitted from the black hole, one finds the characteristic dimensionless ratio [2-5]

$\eta \equiv \frac{\tau_{\text {gap }}}{\tau_{\text {emission }}}=O\left(10^{2}\right)$

\footnotetext{
${ }^{1}$ It is important to emphasize that the black-hole thermal spectrum is distorted by the curvature potential which surrounds the black hole, see Eq. (4) below.
}

a e-mail: shaharhod@gmail.com for Schwarzschild black holes. ${ }^{2}$

The relation (1) implies that the semi-classical radiation flux of Schwarzschild black holes is indeed sparse. Namely, the average time gap between the emissions of successive Hawking quanta out of a Schwarzschild black hole is very large on the timescale $2 \pi / \omega$ [see Eq. (8) below] set by the characteristic energy (frequency) of these emitted quanta. Thus, one may safely conclude that the Hawking quanta of an evaporating Schwarzschild black hole are typically emitted from the black hole one at a time [2-5].

It was also claimed in [2] that adding angular momentum to the black hole makes the dimensionless ratio $\eta$ even larger, thus making the Hawking emission spectra of rotating Kerr black holes even more sparse than the corresponding emission spectrum (1) of the non-rotating Schwarzschild black hole. Namely, it was claimed in [2] that

$\eta_{\text {Kerr }} \geq \eta_{\text {Schwarzschild }}$

As we shall show in this paper, the claim (2) of [2] is actually erroneous. In particular, explicit calculations to be carried below reveal that, for rapidly-rotating Kerr black holes, $\eta(\bar{a})$ is a decreasing function of the dimensionless black-hole angular momentum $\bar{a} .{ }^{3}$ Moreover, as we shall show below, near-extremal Kerr black holes are actually characterized by the relation $\eta(\bar{a} \rightarrow 1)=O(1)$.

\section{The Hawking evaporation process of rapidly-rotating Kerr black holes}

We study the Hawking emission of gravitational quanta by rotating Kerr black holes. The Bekenstein-Hawking temper-

\footnotetext{
${ }^{2}$ Note that for an astrophysical Schwarzschild black hole of ten solar masses, one finds the typical time scale $t_{\text {gap }} \sim 5 \mathrm{~s}$ for the emission of gravitons. For a super-massive Schwarzschild black hole of $10^{7}$ solar masses, one finds the typical time scale $t_{\text {gap }} \sim 5 \times 10^{6} \mathrm{~s}$ for gravitons.

${ }^{3}$ Here $\bar{a} \equiv J / M^{2}$ is the dimensionless angular momentum of the Kerr black hole.
} 
ature of a Kerr black hole and the angular velocity of its horizon are respectively given by the relations ${ }^{4}$

$T_{\mathrm{BH}}=\frac{\hbar\left(r_{+}-r_{-}\right)}{4 \pi\left(r_{+}^{2}+a^{2}\right)} \quad$ and $\quad \Omega_{\mathrm{H}}=\frac{a}{2 M r_{+}}$,

where $r_{ \pm}=M \pm\left(M^{2}-a^{2}\right)^{1 / 2}$ are the (inner and outer) horizon radii of the black hole.

The Hawking emission rate (that is, the number of quanta emitted per unit of time) out of a rotating Kerr black hole is given by the Hawking relation $[1,6,7]$

$\dot{\mathcal{N}} \equiv \frac{d N}{d t}=\frac{\hbar}{2 \pi} \sum_{l, m} \int_{0}^{\infty} d \omega \frac{\Gamma}{e^{x}-1}$,

where $x \equiv \hbar\left(\omega-m \Omega_{\mathrm{H}}\right) / T_{\mathrm{BH}}$. Here $l$ and $m$ (with $l \geq$ $|m|)$ are respectively the spheroidal harmonic index and the azimuthal harmonic index of the emitted quanta, and $\Gamma=\Gamma_{l m}(\omega ; \bar{a})$ are the frequency-dependent gray-body factors $[6,7]$. These dimensionless absorption probabilities quantify the imprint of passage of the emitted black-hole quanta through the effective curvature potential which characterizes the black-hole spacetime.

The Hawking emission rate $\dot{\mathcal{N}}(\bar{a})$ [see Eq. (4)] can be computed along the lines of the numerical procedure described in $[6,7]$. In particular, one finds that, for rapidlyrotating Kerr black holes, the Hawking emission spectrum is greatly dominated by gravitational quanta with the angular indices $[6,7]^{5}$,

$l=m=s=2$.

Moreover, the characteristic thermal factor that appears in the denominator of (4) implies that, for rapidly-rotating (near-extremal, $T_{\mathrm{BH}} \rightarrow 0$ ) black holes, the emission of high energy quanta with $\omega>m \Omega_{\mathrm{H}}$ is exponentially suppressed. Thus, for rapidly-rotating Kerr black holes, the Hawking emission spectra are effectively restricted to the regime

$0 \leq \omega \lesssim m \Omega_{\mathrm{H}}+O\left(T_{\mathrm{BH}} / \hbar\right)$

The reciprocal of the black-hole emission rate,

$\tau_{\text {gap }}=\frac{1}{\dot{\mathcal{N}}}$

determines the average time gap between the emissions of successive Hawking quanta. On the other hand, the characteristic timescale required for each individual Hawking quantum to be emitted from the black hole, $\tau_{\text {emission}}$, can be bounded from below by the time-period it takes to the corresponding emitted wave field to complete a full oscillation

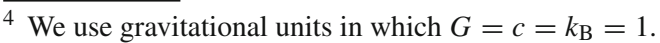

5 The last equality in (5) refers to gravitational quanta, which are characterized by the spin parameter $s=2$.
}

Table 1 The characteristic dimensionless ratio $\tau_{\text {gap }} / \tau_{\text {oscillation of }}$ rapidly-rotating Kerr black holes. Here $\tau_{\text {gap }}$ is the average time gap between the emissions of successive Hawking quanta [see Eq. (7)] and $\tau_{\text {oscillation }}$ is the characteristic oscillation period of the emitted wave field [see Eq. (8)]. One finds that $\eta(\bar{a})$ is a decreasing function of the dimensionless black-hole angular momentum $\bar{a}$. In particular, near-extremal Kerr black holes are characterized by the relation $\eta(\bar{a} \rightarrow 1)=O(1)$

\begin{tabular}{llllll}
\hline $\bar{a} \equiv J / M^{2}$ & 0.90 & 0.96 & 0.99 & 0.999 & 1.0 \\
$\tau_{\text {gap }} / \tau_{\text {oscillation }}$ & 13.5 & 5.5 & 2.5 & 1.5 & 1.2 \\
\hline
\end{tabular}

cycle. That is, ${ }^{6}$

$\tau_{\text {emission }} \geq \tau_{\text {oscillation }}=\frac{2 \pi}{\bar{\omega}}$,

where $\bar{\omega}$ is the characteristic (average) frequency of the emitted Hawking quanta. ${ }^{7,8}$

In Table 1 we display the characteristic dimensionless ratio $\tau_{\text {gap }} / \tau_{\text {oscillation }}$ for rapidly-rotating Kerr black holes. ${ }^{9}$ One finds that $\eta(\bar{a})$ is a decreasing function of the dimensionless black-hole angular momentum $\bar{a}$. In particular, we find that near-extremal Kerr black holes are characterized by the relation ${ }^{10}$

$\eta(\bar{a} \rightarrow 1)=O(1)$

\section{Summary}

It has long been known [2-5] that the Hawking radiation flux out of Schwarzschild black holes is extremely sparse. In particular, these spherically-symmetric black holes are characterized by the large dimensionless ratio $\eta \equiv \tau_{\text {gap }} / \tau_{\text {emission }}=$

\footnotetext{
${ }^{6}$ Note that there is a factor $2 \pi$ difference between our definition of $\tau_{\text {oscillation }}$ and the corresponding definition used in [2]. We believe that the time period $2 \pi / \omega$ required by the emitted wave field to complete a full oscillation cycle [see Eq. (8)] provides a natural lower bound on the characteristic emission timescale $\tau_{\text {emission }}$ of the corresponding Hawking quantum.

7 One finds that for rapidly-rotating Kerr black holes the average frequency of the emitted Hawking quanta is an increasing function of the black-hole angular momentum. Thus, the characteristic emission time [see Eq. (8)] is a decreasing function of the black-hole angular momentum $\bar{a}$.

${ }^{8}$ Using in (8) the peak frequency $\omega_{\text {peak }}$ of the Hawking emission spectrum instead of the average frequency $\bar{\omega}$ of the Hawking emission spectrum would merely change the dimensionless ratio $\eta$ by a factor of order unity.

${ }^{9}$ It is worth emphasizing again that this ratio provides an upper bound on the actual value of $\eta(\bar{a}) \equiv \tau_{\text {gap }} / \tau_{\text {emission, }}$, see Eq. (8).

10 Note that for an astrophysical black hole of ten solar masses and $\bar{a}=0.99$, one finds the typical time scale $t_{\text {gap }} \sim 10^{-3} \mathrm{~s}$. For a supermassive black hole of $10^{7}$ solar masses and $\bar{a}=0.99$, one finds the typical time scale $t_{\text {gap }} \sim 10^{3} \mathrm{~s}$.
} 
$O\left(10^{2}\right)$ [see Eq. (1)]. As recently pointed out in [2], this relation implies that the individual Hawking quanta emitted from a Schwarzschild black hole are well separated in time.

It was recently claimed in [2] that adding angular momentum to the emitting black hole makes the dimensionless ratio $\eta$ even larger [see Eq. (2)], thus making the Hawking radiation spectra of rotating Kerr black holes even sparser than the corresponding emission spectrum of the (non-rotating) Schwarzschild black hole.

In this brief report we have explicitly shown that the claim (2) made in [2] is actually erroneous. In particular, explicit calculations reveal that, for rapidly-rotating Kerr black holes, $\eta(\bar{a})$ is actually a decreasing function of the dimensionless black-hole angular momentum $\bar{a}$. Moreover, we have shown that near-extremal Kerr black holes are characterized by the relation $\eta(\bar{a} \rightarrow 1)=O(1)$.

The relation $\tau_{\text {gap }} / \tau_{\text {emission }}=O(1)$ [see Eq. (9)] implies that the Hawking cascade of gravitons from rapidly rotating Kerr black holes has an almost continuous character. Stated in a more picturesque way, we can say that, on average, there is a gravitational quantum leaving the (rapidly-rotating) black hole at any given moment of time.
Acknowledgments This research is supported by the Carmel Science Foundation. I thank Yael Oren, Arbel M. Ongo, Ayelet B. Lata, and Alona B. Tea for stimulating discussions.

Open Access This article is distributed under the terms of the Creative Commons Attribution 4.0 International License (http://creativecomm ons.org/licenses/by/4.0/), which permits unrestricted use, distribution, and reproduction in any medium, provided you give appropriate credit to the original author(s) and the source, provide a link to the Creative Commons license, and indicate if changes were made. Funded by SCOAP ${ }^{3}$.

\section{References}

1. S.W. Hawking, Commun. Math. Phys. 43, 199 (1975)

2. F. Gray, S. Schuster, A. Van-Brunt, M. Visser, e-print. arXiv: 1506.03975

3. J.D. Bekenstein, V.F. Mukhanov, Phys. Lett. B 360, 7 (1995)

4. J. Mäkelä, Phys. Lett. B 390, 115 (1997)

5. S. Hod, Phys. Lett. A 299, 144 (2002). arXiv:gr-qc/0012076

6. D.N. Page, Phys. Rev. D 13, 198 (1976)

7. D.N. Page, Phys. Rev. D 14, 3260 (1976) 